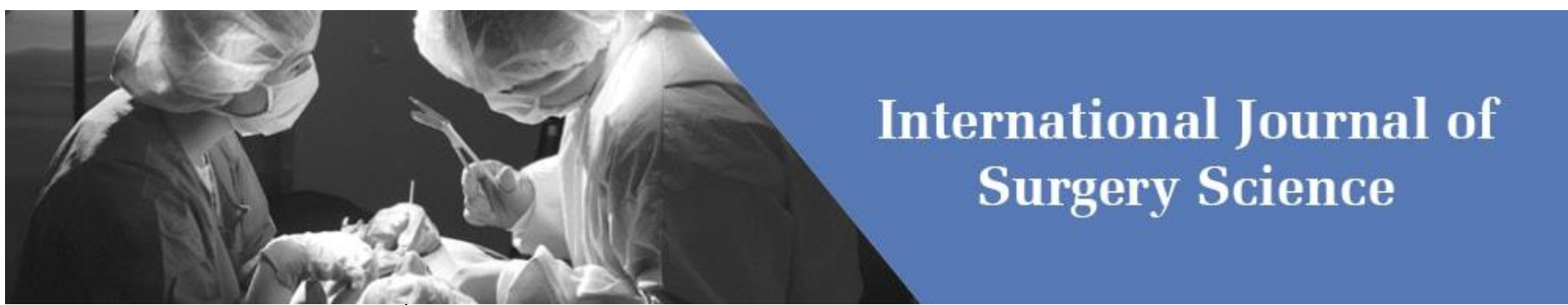

E-ISSN: 2616-3470

P-ISSN: 2616-3462

(C) Surgery Science

www.surgeryscience.com

$2019 ; 3(3): 355-358$

Received: 25-05-2019

Accepted: 27-06-2019

Dr. Joshua Victor

Dept. of General Surgery,

Christian Medical College and

Hospital, Ludhiana, Punjab, India

Dr. Amit Mahajan

Dept. of General Surgery,

Christian Medical College and

Hospital, Ludhiana, Punjab, India

Dr. Anil Luther

Dept. of General Surgery,

Christian Medical College and

Hospital, Ludhiana, Punjab, India

Correspondence

Dr. Amit Mahajan

Dept. of General Surgery,

Christian Medical College and

Hospital, Ludhiana, Punjab, India

\section{A study of correlation of d- dimer with severity of peripheral arterial disease in a tertiary care center of North India: Original research article}

\author{
Dr. Joshua Victor, Dr. Amit Mahajan and Dr. Anil Luther
}

DOI: https://doi.org/10.33545/surgery.2019.v3.i3f.195

\section{Abstract}

Background: Peripheral Arterial Disease is used to describe the impairment of blood flow to extremities as a result of atherosclerotic occlusive disease. Patients with vascular disease have a high risk of mortality, primarily from cardiovascular disease. D-dimer is a product of fibrin degradation by plasmin. Elevated levels of D-dimers are usually considered as marker of increased clotting activity, increased risk of severe atherosclerosis and increased risk of vascular complications.

Aims: To study the correlation of D-dimer with the severity of peripheral vascular disease.

Material and Methods: A prospective study was conducted in the General Surgery Department to study the correlation of D-dimer with peripheral arterial disease. Patients with ankle brachial index $<0.9$ were identified and investigated for D-dimer levels in their blood.

Statistical Analysis: Categorical variables were presented in number and percentage (\%) and continuous variables were presented as mean $\pm \mathrm{SD}$ and median. Normality of data was tested by Kolmogorov-Smirnov test. If the normality was rejected then non-parametric test was used.

Results: A total of 50 patients were included in the study. The study of the demographic details of patients showed increased severity of PAD with increasing age and a male preponderance. Smoking as a risk factor with severity of PAD and diabetes was found to be the most frequently associated co morbidity with PAD. However, no positive correlation could be found between D-dimer levels and co- morbidities, diabetes, hypertension, CAD, CVD and renal disease. D-dimer levels did not correlate with the severity of PAD.

Conclusion: A similar study needs to be done on a larger scale with a more sensitive quantitative estimation of D-dimer levels to yield statistically significant results correlating D- dimer levels with the severity of PAD.

Keywords: D-dimer, atherosclerosis, peripheral arterial disease, ankle brachial index

\section{Introduction}

Peripheral arterial disease (PAD) is atherosclerosis leading to narrowing of the major arteries distal to the aortic arch. Progressive occlusion results in arterial stenosis, reduced blood flow, and claudication, the most common presenting symptom. The most common presenting symptom is claudication; however, only $10 \%$ of patients have classic claudication ${ }^{[1]}$. The measurement of blood pressure at the ankle was proposed as a test for PAD as early as 1950 [2] and led to the development of the ankle-brachial index (ABI). ABI has now become to be the most practical means of demonstrating the presence and severity of PAD. Then many studies were done to study the correlation of biomarkers with the severity of PAD. D-dimer is one such biomarker. The D-dimer antigen is a unique marker of fibrin degradation. D-dimer antigen is the most frequently used indicator of blood coagulation activation. The first studies on the use of Ddimer antigen assays for exclusion of venous thrombosis were published in $1987^{[3]}$. The relationship between blood coagulation and atherogenesis has been studied and proven that hypercoagulability tends to increase atherosclerosis.

Fibrinolysis of fibrin results into degradation products such as the d- dimers. Although D-dimer fragments can be considered to be a marker of fibrinolysis, degradation of fibrin cannot occur without preceding thrombin generation. D-dimers are commonly assessed as markers of ongoing thrombin generation. In general, the plasma levels of these markers are elevated in relation to the extent of atherosclerosis, and have been predictive of cardiovascular events [4]. A pathophysiological explanation for the association between high plasma D-dimer levels and the 
Increased risk of arterial thrombotic events is that D-dimer is part of the so-called inflammation-coagulation-axis. It has been suggested that the generation of several haemostatic enzymes such as thrombin and the increase in fibrin contribute to atherosclerosis progression and modulation of the plaque phenotype. It may be assumed that more the vascular burden of fibrin greater are the amount of fibrin cleavage fragments, due to ongoing fibrinolysis, in patients with extensive atherosclerosis ${ }^{[5]}$ with an increased severity of PAD. Therefore, raised D-dimer levels are associated with severe PAD. Since there were very few studies in the Indian population to correlate the levels of Ddimer with the severity of PAD, we conducted this study.

\section{Aim and Objective}

To study the correlation of D-dimer with severity of peripheral arterial disease.

\section{Material and Methods \\ Study Design}

A prospective observational study was conducted in the General Surgery department of tertiary care center of North India to study the correlation of D-dimer with severity of peripheral arterial disease. The study was done after taking necessary permissions from the hospital ethics committee. All consecutive patients reporting to our tertiary care center satisfying the inclusion criteria were included in the study Patients with ankle brachial index $<0.9$ were identified and investigated for d-dimer levels in their blood. Data was collected from all the consecutive patients admitted in General Surgery department over a period of one and half years (from $15^{\text {th }}$ December 2015 to $14^{\text {th }}$ June 2017).

\section{Inclusion Criteria}

All consecutive patients admitted in the General Surgery department with symptoms suggestive of peripheral arterial disease such as claudication pain, rest pain and ankle brachial index $<0.9$ were taken for the study.

\section{Exclusion Criteria}

1. Patients with $A B I \geq 0.9$ were excluded.

2. Patients with bleeding disorders, coagulopathy, DVT.

3. Patients who did not give consent for study were excluded.

\section{Methodology \\ ABI measurement}

The ankle-brachial index (ABI) is a simple, noninvasive tool used to screen for peripheral arterial disease (PAD. Measurement of the ABI can be easily performed using a blood pressure (BP) cuff and handheld Doppler device Bistoshidop 8 Mhz. Systolic BP is determined in both arms and both ankles.

We then divided the higher of the two systolic pressures for each leg by the higher of the two arm pressures to get the right and left ABI.

Table 1: Interpretation of ABPI with respect to disease

\begin{tabular}{|c|c|}
\hline Ankle Brachial Index report & Interpretation \\
\hline$\leq 0.9$ & Peripheral arterial disease \\
\hline 0.91 to 0.99 & Borderline \\
\hline $1.0-1.4$ & Normal \\
\hline$>1.4$ & Non compressible arteries \\
\hline
\end{tabular}

On the basis of ABI patients were further classified as having mild, moderate or severe PAD as follows:
Table 2: Criteria used to decide the severity of PAD in study

\begin{tabular}{|c|c|}
\hline Ankle Brachial Index report & Interpretation \\
\hline 0.71 to 0.90 & Mild peripheral arterial disease \\
\hline 0.41 to.70 & Moderate peripheral arterial disease \\
\hline$<0.40$ & Severe peripheral arterial disease \\
\hline
\end{tabular}

\section{Blood-collection}

Two ml EDTA plasma specimens were collected.

\section{D-dimer levels}

Tulip XL FDP KIT was used. XL FDP slide test for detection of cross linked fibrin degradation products is based on the principle of agglutination. The test specimen (plasma) was mixed with XL FDP latex reagent. The sensitivity of the reagent is $\approx 200 \mathrm{ng} / \mathrm{ml}$, below which samples are negative and above which samples give a positive agglutination reaction. The time taken for agglutination to occur was noted and interpreted as follows ${ }^{[6]}$.

Table 3: Time taken for agglutination to occur

\begin{tabular}{|c|c|}
\hline D-dimer report & Interpretation \\
\hline Absent & No agglutination \\
\hline $1+$ & $1 \mathrm{~min} 30 \mathrm{sec}-2 \mathrm{~min}$ \\
\hline $2+$ & $1 \mathrm{~min}-1 \mathrm{~min} 30 \mathrm{sec}$ \\
\hline $3+$ & $30 \mathrm{sec}-1 \mathrm{~min}$ \\
\hline $4+$ & $0-30 \mathrm{sec}$ \\
\hline
\end{tabular}

\section{Sample Size}

On the basis of previous study, prevalence of PAD in adult population was $12 \%$. Taking this value as reference, the minimum required sample size with $10 \%$ margin of error and $5 \%$ level of significance is 41 patients. Hence the total sample size taken was 50 .

Formula used was:-

$\mathrm{N} \geq\left(\left(\mathrm{p}(1-\mathrm{p})\left(\mathrm{ME} / \mathrm{z}_{\alpha}\right)^{2}\right.\right.$

Where $\mathrm{Z}_{\alpha}$ is value of $\mathrm{Z}$ at two sided alpha error of $5 \%, \mathrm{ME}$ is margin of error and $\mathrm{p}$ is prevalence rate.

\section{Statistical Analysis}

Statistical tests were applied as follows-

1. Quantitative variables were compared using ANOVA/ Kruskalwallis test (when the data sets were not normally distributed) between the three groups.

2. Qualitative variables were correlated using Chi-Square test /Fisher's exact test.

3. Univariate and multivariate ordinal regression was used to assess the association of parameters with severity.

A p value of $<0.05$ was considered statistically significant.

The data was entered in MS EXCEL spreadsheet and analysis was done using Statistical Package for Social Sciences (SPSS) version 21.0.

\section{Informed Consent}

All patients were informed about the purpose of study prior to enrollment and written consent was taken.

\section{Result and Analysis}

This was a prospective study comprises of 50 patients of either sex 18 years of age or more who presented with peripheral arterial occlusive disease. Patients were assessed on the basis of history, clinical examination and relevant investigations done were noted in the data sheet according to the proforma.

The data was collected, analysed and the results are as follows: 
Age: The peak incidence of PAD is found to be in the age group from 51-60 years. $32 \%$ were in the age group 51 to $60 \mathrm{yrs}, 28 \%$ aged $61-70 \%$ and $16 \%$ aged $>70$ yrs. Patients in the age group between $61-70$ years tend to have more severe disease. Criqui et $a l$. showed that the prevalence of PAD was $5.6 \%$ in persons aged 51 to 60 years, $15.9 \%$ in persons aged 61 to 70 years old, and $33.8 \%$ in persons aged $>70$ years old ${ }^{[7]}$ Hager et al. showed high D-dimer levels in elderly subjects ${ }^{[8]}$. But in our study there here was no statistically significant $(\mathrm{p}=0.923)$ correlation between age of the patient and dimer levels

\section{Gender}

In our study out of the total 50 patients, there were 40 males and 10 females with females comprising 20\%. A similar male: female ratio of 4:1 was shown in a study by Schroll et al., ${ }^{[9]} \mathrm{D}$ dimer showed a trend towards statistical difference by gender (median value 188for men and 210 for women, $p$ 5. 04 for levels of d-dimer) in a study by Pieper et al., ${ }^{[10]}$ This was contradictory to our study which did not show a statistically significant correlation.

\section{Risk factors for PAD \\ Smoking}

Smoking is found to be major risk in our patients with PAD. Out of 50 patients 21 patients were smokers comprising $42 \%$ of the study population. Among the smokers $45.83 \%$ had severe disease. Smoking is one of the strongest risk factors for PAD in virtually all studies. In a study by Joosten et al. $44 \%$ of smokers had PAD ${ }^{[11]}$. This is comparable to the results of our study.

\section{Diabetes Mellitus}

In our study diabetes mellitus was a significant risk factor found to be associated with $56 \%$ of our patients. Out of 50 patients, 28 patients had diabetes mellitus. In NHANES, $26 \%$ of participants with PAD were identified as having diabetes ${ }^{[12]}$.

\section{Hypertension}

Our study also found a strong association of hypertension with PAD. Out of 50 patients, 24 patients (48\%) had systemic hypertension. Almost every study has shown a strong association between hypertension and PAD, and as many as $50 \%$ of patients with PAD had hypertension in a study by Olin et al., ${ }^{[13]}$

Table 4: Correlation of D-dimer with severity of PAD

\begin{tabular}{|c|c|c|c|c|c|}
\hline \multirow{2}{*}{ D-Dimer } & \multicolumn{3}{|c|}{ Severity } & \multirow{2}{*}{ Total } & \multirow{2}{*}{ P Value } \\
\cline { 2 - 4 } & Mild & Moderate & Severe & & \\
\hline $1+$ & $4(19.05 \%)$ & $1(20.00 \%)$ & $4(16.67 \%)$ & $9(18.00 \%)$ & \\
$2+$ & $8(38.10 \%)$ & $3(60.00 \%)$ & $11(45.83 \%)$ & $22(44.00 \%)$ & \\
$3+$ & $3(14.29 \%)$ & $0(0.00 \%)$ & $5(20.83 \%)$ & $8(16.00 \%)$ & 0.853 \\
$4+$ & $2(9.52 \%)$ & $1(20.00 \%)$ & $1(4.17 \%)$ & $4(8.00 \%)$ & \\
Negative & $4(19.05 \%)$ & $0(0.00 \%)$ & $3(12.50 \%)$ & $7(14.00 \%)$ & \\
Total & $21(100.00 \%)$ & $5(100.00 \%)$ & $24(100.00 \%)$ & $50(100.00 \%)$ & \\
\hline
\end{tabular}

There was no statistically significant $(\mathrm{p}=0.835)$ correlation between ankle brachial index and D-Dimer levels.

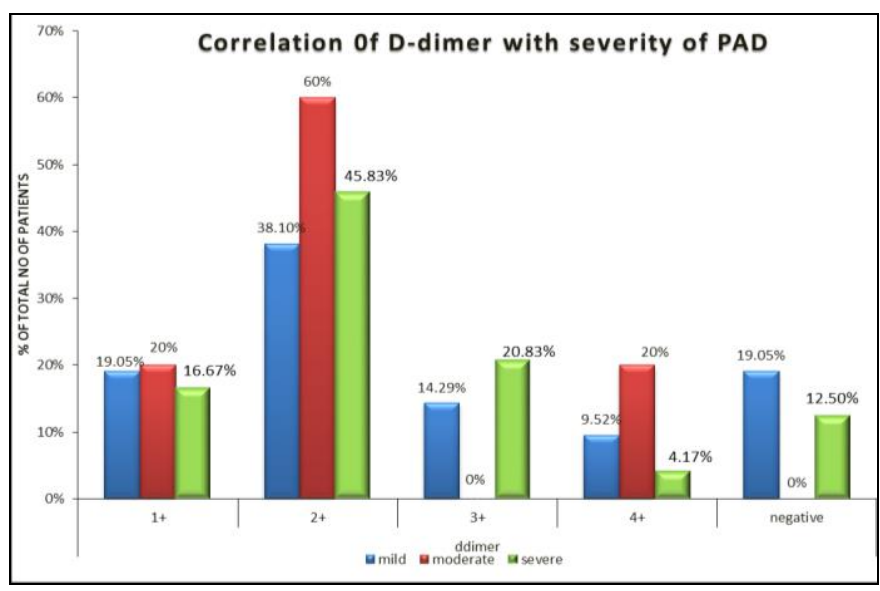

Fig 1: Correlation of D-dimer with severity of PAD

\section{Discussion}

Peripheral artery disease (PAD), which comprises atherosclerosis of the abdominal aorta, iliac, and lowerextremity arteries, is under diagnosed and undertreated. Patients with PAD may present with problems, such as claudication, ischemic rest pain, ischemic ulcerations, repeated hospitalizations, revascularizations, and limb loss. This may lead to a poor quality of life.

In this prospective prevalence study conducted in patients seeking care at our medical college for peripheral arterial disease, we aimed at determining the correlation of D-dimer with severity of peripheral arterial disease of lower limb. A total of 50 patients were included in the study. Patients were assessed on the basis of history, clinical examination and relevant investigations done were noted in the data sheet. Ankle Brachial Index was calculated for each leg. A blood sample was drawn and D-dimer levels were checked using a latex agglutination test. D-Dimer and co-relation was done with the severity of PAD.

D-dimer has been shown to be elevated in patients with peripheral arterial disease. D-dimer levels have also been shown to relate to the severity of the atherosclerosis ${ }^{14}$. Elevated levels of D-dimers are usually considered as a marker of increased clotting activity. This assumption is one of the key elements of the controversy regarding cause and consequence of hypercoagulability. Indeed, Herren et al. observed increased levels of D-dimer in patients with PAD, correlating with severity of disease ${ }^{15}$. The first study that assessed the value of D-dimer as an independent predictor for arterial thrombotic events was performed in 1993 by Fowkes et al., ${ }^{[16]}$ An elevated D-dimer has been associated with a lower ABI ${ }^{[17]}$. Increased fibrin Ddimer reflecting an overall increased coagulation has been demonstrated in patients with PAD ${ }^{[18]}$. A study by Fowkes et al. had suggested that D-dimer was independently associated with a reduction in $\mathrm{ABI}^{16}$. There are very few studies done in Indian population to correlate D-dimer levels with severity of PAD. Hence we undertook this study. However in our study there is no significant correlation between D-dimer and the severity of PAD as shown by ankle brachial index. A similar study by Buyzere $e t$ al. also failed to show a significant correlation between D-dimer and the severity of PAD ${ }^{[19]}$. Among our study population there was a significant coexistence of comorbidities, 28 patients were diabetics, 24 were hypertensive and one patient had CVD. The multiple medications taken by these patients could have 
confounded the correlation of d-dimer levels with severity of PAD. According to a study by Kocas et al. Latex agglutination assay (semi-quantitative) D-dimer analysis is less specific than quantitative analysis in diagnosing pulmonary thromboembolism ${ }^{[20]}$. Hence a similar limitation due to the use of the less sensitive semi quantitative assay can also be a reason for failure of correlation of the D-dimer levels with severity of PAD.

A study would have to be conducted in a larger population and D-Dimer levels will have to be estimated using the more sensitive quantitative assays to establish a better correlation between D-dimer levels and the severity of PAD.

\section{Conclusion}

Our study done among 50 patients diagnosed with PAD aimed to study the correlation of D-dimer levels with the severity of PAD. We summarize our conclusions as follows that the study of the demographic details of patients showed increased severity of PAD with increasing age and a male preponderance. We found a strong association of smoking as a risk factor with severity of PAD and diabetes was found to be the most frequently associated co morbidity with PAD. However no positive correlation could be found between D-dimer levels and the co- morbidities, diabetes, hypertension, CAD, CVD and renal disease. D-dimer levels did not correlate with the severity of PAD. A similar study would have to be done on a larger scale with a more sensitive quantitative estimation of D-dimer levels to yield statistically significant results correlating D- dimer levels with the severity of PAD. The study population should not have co- morbidities to avoid confounding D-dimer levels by the medications taken by these patients to treat their comorbidities.

\section{References}

1. Hirsch AT, Criqui MH, Treat-Jacobson D, Regensteiner JG, Creager MA, Olin JW et al. Peripheral arterial disease detection, awareness and treatment in primary care. Jama. 2001; 286:1317-24.

2. Winsor T. Influence of arterial disease on the systolic blood pressure gradients of the extremity. Am J Med Sci. 1950; 220:117-26.

3. Hillyard CJ, Blake AS, Wilson K, Rylatt DB, Miles S, Bunch RA. Latex agglutination assay for $\mathrm{D}$ dimer: evaluation and application to the diagnosis of thrombotic disease. Clin Chem. 1987; 33:1837-40.

4. Foley CJ, Nichols L, Jeong K, Moore CG, Ragni MV. Coronary atherosclerosis and cardiovascular mortality in hemophilia. J Throm Haemost. 2010; 8:208-11.

5. Ridker PM, Hennekens CH, Cerskus A, Stampfer MJ. Plasma concentration of crosslinked fibrin degradation product (D-dimer) and the risk of future myocardial infarction among apparently healthy men. Circulation. 1994; 90:2236-40.

6. Pangana KD, Pangana TJ. Mosby's diagnostic and laboratory test reference, $2^{\text {nd }}$ edition, USA. Mosby. 1995; 297-8.

7. Criqui MH, Fronek AM, Barrett-Connor E, Klauber MR, Gabriel S, Goodman D. The prevalence of peripheral arterial disease in a defined population. Circulation. 1985; 71:510-5.

8. Hager K, Platt D. Fibrin degeneration product concentrations (D-dimers) in the course of aging. Gerontology. 1995; 41:159-65.

9. Schroll M, Munck O. Estimation of peripheral arteriosclerotic disease by ankle blood pressure measurements in a population of 60 year old men and women. J Chronic Dis. 1981; 34:261-9.

10. Pieper CF, Rao KM, Currie MS, Harris TB, Cohen HJ. Age, functional status, and racial differences in plasma D-dimer levels in community-dwelling elderly persons. J Gerontol A BiolSci Med Sci. 2000; 55:649-57.

11. Hager K, Platt D. Fibrin degeneration product concentrations (D-dimers) in the course of aging. Gerontology. 1995; 41:159-65.

12. Joosten MM, Pai JK, Bertoia ML, Rimm EB, Spiegelman $\mathrm{D}$, Mittleman MA et al. Associations between conventional cardiovascular risk factors and risk of peripheral artery disease in men. Jama. 2012; 308:1660-7.

13. Selvin E, Hirsch AT. Contemporary risk factor control and walking dysfunction in individuals with peripheral arterial disease: NHANES. Atherosclerosis. 2008; 201:425-33.

14. Olin JW. Hypertension and peripheral arterial disease. Vasc Med. 2005; 10:241-6.

15. Van der Bom JG, Bots ML, Haverkate F, Meijer P, Hofman A, Kluft $\mathrm{C}$ et al. Activation products of the haemostatic system in coronary, cerebrovascular and peripheral arterial disease. Throm Haemost. 2001; 85:234-39.

16. Herren T, Stricker H, Haeberli A, Do DD, Straub PW. Fibrin formation and degradation in patients with arteriosclerotic disease. Circulation 1994; 90:2679-86.

17. Fowkes FG, Lowe GD, Housley E, Rattray A, Rumley A, Elton RA et al. Cross-linked fibrin degradation products, progression of peripheral arterial disease, and risk of coronary heart disease. Lancet. 1993; 342:84-6.

18. Unlu Y, Karapolat S, Karaca Y, Kiziltunc A. Comparison of levels of inflammatory markers and hemostatic factors in the patients with and without peripheral arterial disease. Thromb Res. 2006; 117:357-64.

19. Cortellaro M, Cofrancesco E, Boschetti C, Mussoni L, Donati MB, Catalano $\mathrm{M}$ et al. Association of increased fibrin turnover and defective fibrinolytic capacity with leg atherosclerosis. The PLAT Group. Thromb Haemost 1994; 72:292-6.

20. De Buyzere MA, Philippé J, Duprez D, Baele G, Clement DL. Coagulation system activation and increase of D-dimer levels in peripheral arterial occlusive disease. Am J Hematol. 1993; 43:91-4.

21. Kovacs MJ, MacKinnon KM, Anderson D, O'rourke K, Keeney M, Kearon C et al. A comparison of three rapid Ddimer methods for the diagnosis of venous thromboembolism. Br J Haematol. 2001; 115:140-4. 